\title{
Evolution of Rpp8 genes in Arabidopsis heynh: in Holl \& heynh
}

\begin{abstract}
Rpp8 is present in Arabidopsis chromosomes in two different configurations: either as a tandem duplication with the loci separated by approximately $1 \mathrm{~kb}$, or as a single-copy gene. Polymorphism in gene copy numbers is common in clusters, and is also present in single-copy loci as the presence or absence of a locus. Copy number dynamics, intergenic exchange, and allelic diversity are all likely to be evolutionary responses to the same selective pressures for disease resistance. R-gene evolution, therefore, has both a vertical component across generations and a horizontal component throughout the genome, and each is likely to be shaped by natural selection for resistance. The presence of ancient and many segregating alleles at R-gene loci is interesting because disease resistance is thought to involve an evolutionary arms race between host and pathogen. Because of the lack of research on the polymorphism, this paper will investigate the polymorphism in each of the three loci, and determine how much of it is shared between the loci. From this, we can determine whether there is evidence for interlocus exchange, and if so, whether intergenic exchange is more common between the two members on the same chromosome (intra-chromosomal exchange), or whether exchange occurs more often between chromosomes. This paper also investigates whether these loci harbor more nucleotide polymorphism than that at another locus, which for the purpose of this lab can be considered to have "typical" levels of polymorphism for the species. From the neighbor-joined phylogeny, there is a significant amount of evidence for interlocus exchange. Around $\sim 5 \%$ of the segregated DNA polymorphism is shared between loci and $95 \%$ is distinct to each locus. There is evidence for interlocus exchange. Intergenic exchange is more common between B and C loci than it is between any other loci combination. There is elevated polymorphism at Rpp8. All of these observations are coming from the hypothesis that the polymorphism demonstrated in this paper is coming from a continuously maintained balance of variation created by new mutations and natural selection. This seems to fit with the context of the gene sequences, as they are coming from Arabidopsis, which is self-fertilizing. Some future directions of research include looking for the cause of the increased polymorphism in Rpp8. More work should be done to clarify this relationship and compare it to similar relationships with other genes. Also more work should be done to find molecular evidence for this relationship.
\end{abstract}

Keywords: phylogenetics, morphology, polymorphism, evolution, biochemistry, bioinfomatics
Volume 3 Issue 4 - 2018

\section{Sammer M Marzouk}

University of Chicago Laboratory Schools, USA

Correspondence: Sammer M Marzouk, University of Chicago Laboratory Schools, I 362 E 59th St, Chicago, IL, USA 60637, Email marzouk.sammer@gmail.com

Received: June II, 2018 | Published: July 05, 2018

\section{Introduction}

The induction of plant defense responses by invading pathogens involves specific interactions between host and pathogen that bear important resemblances to human defenses against disease. These induced defenses mainly consist of multiple physiological responses, including localized cell death (called the hypersensitive response; HR), that result from the rapid phosphorylation of an array of proteins and the induction of genes through several pathways. Classical genetic studies of the HR, most notably for rust resistance in flax, have led to the gene-for-gene hypothesis, where a plant resistance $(R)$ gene confers resistance specific to a corresponding avirulence (avr) gene in a pathogen. ${ }^{1}$ Overwhelming genetic evidence indicates that $\mathrm{R}-$ genes act as receptors for avr-gene products or avr-gene-dependent ligands, and effect changes in downstream gene action. ${ }^{2}$ In plant Rgenes, polymorphism is often associated with loci that are present as tandem arrays of multiple copies. Due to promiscuous genetic exchange, paralogs within these clusters exhibit complex evolutionary relationships. ${ }^{3,4}$ Polymorphism in gene copy numbers is common in clusters, and is also present in single-copy loci as the presence or absence of a locus. ${ }^{5-7}$ Copy number dynamics, intergenic exchange, and allelic diversity are all likely to be evolutionary responses to the same selective pressures for disease resistance. R-gene evolution, therefore, has both a vertical component across generations and a horizontal component throughout the genome, and each is likely to be shaped by natural selection for resistance. ${ }^{8}$ The presence of ancient and many segregating alleles at R-gene loci is interesting because disease resistance is thought to involve an evolutionary arms race between host and pathogen.

A classic arms race is one that entails a series of selective sweeps as novel R-gene alleles, capable of recognizing pathogenecity determinants (called avirulence (Avr) factors) that previously avoided detection in a plant population, spread to high frequency. ${ }^{9}$ Support for these evolutionary dynamics centers on the common observation that amino acids evolve at a faster rate in functionally important regions of Rgene proteins than the corresponding rate of synonymous change. ${ }^{10}$ But according to the population genetics theory of selective sweeps, the rapid turnover of new $\mathrm{R}$-gene specificity should cause a 
reduction in the age and number of alleles at a locus. ${ }^{11} \mathrm{Rpp} 8$ is present in Arabidopsis chromosomes in two different configurations: either as a tandem duplication with the loci separated by approximately $1 \mathrm{~kb}$, or as a single-copy gene. ${ }^{12-14}$ The loci are labeled A, B, and C, respectively. Because of the lack of research on the polymorphism, this paper will investigate the polymorphism in each of the three loci, and determine how much of it is shared between the loci. From this, we can determine whether there is evidence for interlocus exchange, and if so, whether intergenic exchange is more common between the two members on the same chromosome (intra-chromosomal exchange), or whether exchange occurs more often between chromosomes. This paper also investigates whether these loci harbor more nucleotide polymorphism than that at another locus, which for the purpose of this lab can be considered to have "typical" levels of polymorphism for the species. ${ }^{15}$ This evidence will be used to look at the question of whether selection and/or frequent exchange has enhanced polymorphism in the R-genes

\section{Materials and methods}

\section{Gene information}

The focus of this paper will be on the Rpp8 gene. The Rpp8 data is contained in a file called "Rpp8_exon.nex"; it is a nexus file consisting of exon sequences for $6 \mathrm{~A}$ genes, $6 \mathrm{~B}$ genes, and $8 \mathrm{C}$ genes from $\mathrm{A}$. thaliana. There are also two sequences from one chromosome of a related species, $A$. lyrata, labeled $\mathrm{Ce} 4 \mathrm{~B}$ and $\mathrm{Ce} 4 \mathrm{~A}$. The sequences are all aligned. The coding sequence is in frame.

\section{PubMed Entrez}

Entrez is a molecular biology database system that provides integrated access to nucleotide and protein sequence data, genecentered and genomic mapping information, 3D structure data, PubMed MEDLINE, and more. The system is produced by the National Center for Biotechnology Information (NCBI) and is available via the Internet. Entrez covers over 20 databases including the complete protein sequence data from PIR-International, PRF, Swiss-Prot, and PDB and nucleotide sequence data from Gen Bank that includes information from EMBL and DDBJ. The Entrez retrieval system uses an intuitive user interface for rapidly searching sequence and bibliographic data. A unique feature of the system is its use of precomputed similarity searches for each record to create links to "neighbors" or related records in other Entrez databases. These links facilitate integrated access across the various databases. An Entrez global query provides search capability for a subset of Entrez databases at one time. Results may be viewed in various formats including Flat File, FASTA, XML, and others. A graphical interface provides easy visualization of complete genomes or chromosomes, as well as biological annotation on individual sequences. Entrez also allows Batch downloads of large search results. This was used to find sequences of ferulate-5-hydroxylase (FAH1) and A. lyrata (L.) O'Kaneb \& Al-Shehbaz.

\section{HyPhy}

HyPhy (Hypothesis Testing using Phylogenies) is an opensource software package for the analysis of genetic sequences (in particular the inference of natural selection) using techniques in phylogenetics, molecular evolution, and machine learning. ${ }^{1}$ The paper uses this software to compare the independent and dependent phylogenies through the use of a bootstrap analysis. The bootstrap was run within normal and default parameters. In the bootstrap, the minimum number of simulation recommended was 100 . The program allows 100-1000 simulations. For this analysis, 550 simulations were run. The simulations calculated the LR value. The LR value is the likelihood ratio, defined as $2(\log \mathrm{L}-\log \mathrm{L} 0)$, where LA is the likelihood for the alternative hypothesis, L0 is the likelihood for the null hypothesis (refer to the documentation for HyPhy). ${ }^{1}$ A simulation in the bootstrap is to pick random sites from the original sequence with replacement, rebuild the phylogenetic tree for two hypotheses, calculate the log likelihood and generate one likelihood ratio. The goal is to see the likelihood ratio from the data fall into the empirical distribution. In the program, the null hypothesis was entered and the alternative hypothesis. ${ }^{1}$ A null hypothesis supports the hypothesis that there is no significant difference between specified population; that any observed difference being due to sampling or experimental error. The alternative hypothesis supports the hypothesis that there is a significant difference between specified populations and that these differences share a cause. If the $\mathrm{p}-$ value is really small, $\sim \mathrm{p}<0.0005$, the null hypothesis is rejected. The possibility of proximal and distal genes evolving independently as the alternative hypothesis. And the possibility of proximal and distal genes evolving independently as the null hypothesis. ${ }^{1}$ The MEGA software was also used to align and organize the DNA before the phylogenies were created.

\section{DNASP}

DnaSP (DNA Sequence Polymorphism) is a software package for the analysis of DNA polymorphisms using data from a single locus (a multiple sequence aligned-MSA data), or from several loci (a Multiple-MSA data, such as formats generated by some assembler RAD-seq software). DnaSP can estimate several measures of DNA sequence variation within and between populations in noncoding, synonymous or nonsynonymous sites, or in various sorts of codon positions), as well as linkage disequilibrium, recombination, gene flow and gene conversion parameters. Moreover, DnaSP can conduct a number of neutrality tests, such as (among others), the Hudson, Kreitman and Aguadé (1987), Tajima (1989), McDonald and Kreitman (1991), Fu and Li (1993), and Fu (1997), Ramos-Onsins and Rozas, Achaz (2009) tests, and compute their confidence intervals by the coalescent. The results of the analyses are displayed on tabular and graphic form. This was used in the paper in order to calculate the Fisher and G-tests results.

\section{MEGA}

MEGA (Molecular Evolutionary Genetics Analysis) is software that specializes in analyzing FASTA DNA sequences. The software emphasizes the integration of sequence acquisition with evolutionary analysis. It contains an array of input data and multiple results explorers for visual representation; the handling and editing of sequence data, sequence alignments, inferred phylogenetic trees; and estimated evolutionary distances. ${ }^{2}$ The software allows the user the ability to browse, edit, summarize, export, and generate publicationquality captions for their results. MEGA also includes distance matrix and phylogeny explorers as well as advanced graphical modules for the visual representation of input data and output results. The main features of this software used in this paper are the phylogeny construction software and the substitution software. ${ }^{2}$ The substitution software will analyze the DNA sequences that are uploaded onto 
the program, after which, it will calculate the AiC value for each substitution model. The model with the lowest $\mathrm{AiC}$ value will be the model that will be used to analyze that sequence. See the supplementary information for more specifics on the AiC calculation. After the substitution model was determined, a phylogeny would be created with the gathered information. All of the phylogenies created using the Neighbor-Joining method. ${ }^{2}$

\section{Results}

To begin the analysis, a phylogeny was created using the Rpp8 sequences (Table 1). This was a simple phylogeny that was created with neighbor-joining techniques (Figure 1). From the phylogeny, it is demonstrated that there is interlocus exchange between the A, $\mathrm{B}$, and $\mathrm{C}$ loci. In the phylogeny, it is shown that there is significant overlap. This overlap is shown by the general shape of the phylogeny. Each clade of the phylogeny is directly connected to each other clade. In the phylogeny, there is no true outlier group. Every branch shares significant similarities with other branches within and in different clades. Also, the phylogeny is in two clades. There is one "outlier," WUA that shares remarkable similarities with the rest of the gene sequences. And the second clade is one big clade that devolves into smaller clades. This de-evolution of the clade design shows significant amount of genetic overlap between the sequences. And in the second clade, it is shown that almost a third of genetic samples are considered to have the same genetic differences.

Table I Nexus File for Rpp8 Phylogeny

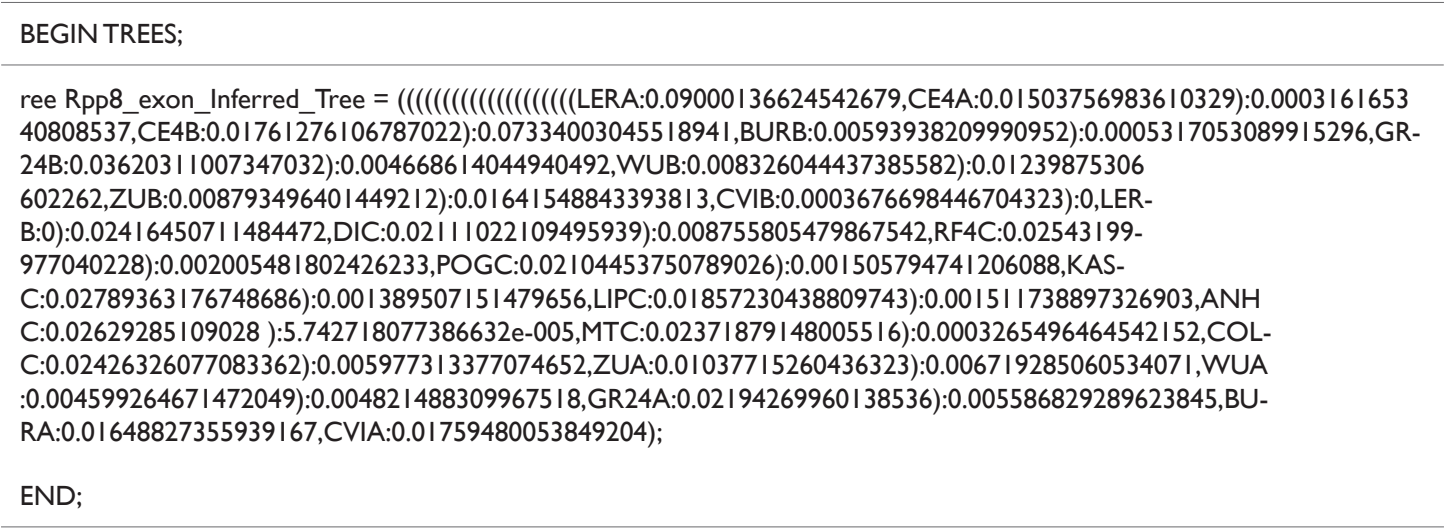

This is a phylogeny in nexus form that was created from the Rpp8 sequences from the MEGA software. It was created using neighbor-joining techniques.

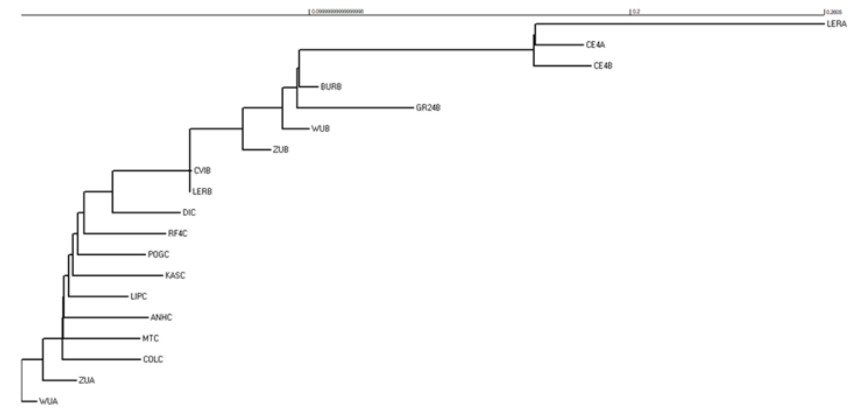

Figure I Phylogeny of Rpp8 Sequences.

This is a phylogeny that was created from the Rpp8 sequences from the MEGA software. It was created using neighbor-joining techniques.

The next step of the analysis was using the DNASP software. Something to note is that the final three bases in the gene sequences is the "stop" codon. This is generally not considered part of the coding region. As such, this paper excludes the last three bases for the purpose of analysis. The codon position of the first site is "1". Five datasets were created: one consisting of the six A. thaliana A locus alleles, the second consisting of the six A. thaliana B (L.) Heynh locus alleles, the third consisting of the eight $\mathrm{A}$. thaliana $\mathrm{C}$ locus alleles, the fourth combining the $20 \mathrm{~A}+\mathrm{B}+\mathrm{C}$ alleles, and the fifth consisting of the two A. lyrata alleles (Ce4A and Ce4B). Polymorphism analysis was conducted of the entire dataset, with the exclusion of the lyrata sequences. From the Polymorphic site test, there were 366 polymorphic sites identified out of 2664 total sites (Table 2). The DNA polymorphism test was also applied on the same set of data with the same restrictions. Pi was calculated to be 0.04005 , $\mathrm{k}$ was 107 , and theta was 103.164.

After the basic divergence test, the DNASP software was then programmed to focus on the individual loci and the ways compare the individual loci. Looking at the A and B divergence, there were 12 sequences with 294 sequence sites and 325 total mutations. The $\mathrm{k}-$ value was $\sim 105$. The pi value was 0.035 (Table 4 ). The Dx/a value were 0.04 and the $\mathrm{Da}$ value is 0.02 . Looking at the $\mathrm{A}$ and $\mathrm{C}$ divergence, there were 14 sequences with 302 polymorphic sites and 352 total mutations. The $\mathrm{k}$-value was $\sim 109$, the pi value was $\sim 0.036$, the $\mathrm{Dx} / \mathrm{a}=0.03665$, and the $\mathrm{Da}$ value is 0.00138 (Table 4). For the third comparison, the B loci were compared to the C loci. There were 14 total sequences with 346 polymorphic sites and 398 total mutations. The $\mathrm{k}$-value was 110.648 and pi was equal to 0.04153 (Table 4). The Dx/y ratio was 0.04576 and the Da ratio was 0.01023 (Table 4). The final step of this analysis was using the DNASP using the HKA calculation method. By comparing the polymorphism within each species and the divergence observed between two species at two or more loci, the test can determine whether the observed difference is likely due to neutral evolution or rather due to adaptive evolution. To use the HKA calculator, we needed to determine how many segregating sites there were in the samples of each of the Rpp8 loci and the sample of the FAH1 alleles. It is already known how many 
segregating sites there were for the Rpp8 loci, so the attention was focused on the FAH1 alleles. The FAH1 alleles were found to have 10 segregating sites from DNA polymorphism testing (Table 5). After this, the amount of data was complete. For the HKA test, we had to calculate the number of differences between one A. lyrata allele and one A. thaliana allele for each of the loci (Rpp8 and FAH1). This was overcome by selecting a random $A$. lyrata gene and then finding its difference from each of the loci (Table 6). For the first locus, there were 159 differences. For the second locus, there were 334 differences (Table 6). This data was then put into the HKA test, which completed the requirements for the calculator. From the HKA-calculator, the $\mathrm{p}$-value was calculated to be 0.0000 , and the $\mathrm{X}$-squared values was found to be 81.945 (Figure 6). Both of the calculations were done relative to the auto some (Table 6).

Table 2 Polymorphism Site Test

\section{Polymorphic sites}

Input Data File: C:1...IRpp8_exon.nex

Population used:A_and_B_and_C

Number of sequences used: 20

Selected region: I-2748 Number of sites: 2748

Number of sites (excluding fixed gaps / missing data): 2742

Total number of sites (excluding sites with gaps / missing data): 2664

Sites with alignment gaps or missing data: 84

Invariable (monomorphic) sites: 2298

Variable (polymorphic) sites: 366 (Total number of mutations: 428)

Singleton variable sites: II

Parsimony informative sites: 249

\section{Singleton variable sites (two variants): 109}

Site positions: 2237 I80 30230535035 I 353357387564695765

$8 \mid 686894$ | 970 I038 I058 I087 || || | |223 |297 |309 |3|7 |323

1346

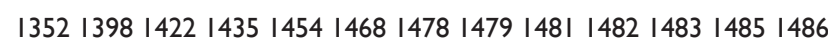
1487

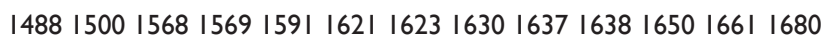
1697

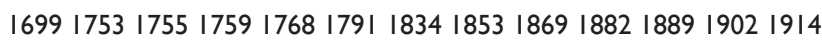
1916

$192|1923| 925|928| 933|944| 975|980200020172038204| 2053$ 2054

2069207820822113213621882189219522002237228622942342 2371

$2436244024422489249|251025172558256| 257526052743$
Table Continued...

Parsimony informative sites (two variants): 200

Site positions: 49 I। I3 27283 I 6779869 I I30 I32

I772592632772953043093I232738I 393422430526

$52753854055956 \mid 56258063665$ I 68 I 738744745747

750767769772774778779792798801802809813817

$8568579 \mid 2928957966974975$ IIII II I26 I| 66 |20| I203 I23।

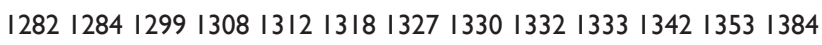
1399

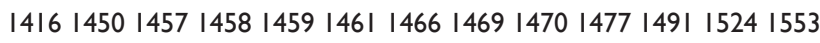
1565

1594 I596 I639 I652 I659 I660 I684 I698 I706 I708 I766 I799 I840 1842

1849190319061910192419301931193819631966197819811982 1984

1994199519971999200820452052207220932098210821102112

2116

2117212321382146216821692187219722042272227322742277 2278

$22842285229|23| 4232523382339234023432344234723502355$ 2362

237024022409242 I 24242425244 I 244524482467246824692472 2476

249024942495249625032506250925 II 25 I 425232573258 I 26 I0 2634

26372645264827042744

Singleton variable sites (three variants): 8

Site positions: 255343 I345 I898 I9I2 I922 23532357

Parsimony informative sites (three variants): 44

Site positions: 2 I 369900 I|43 I|9| I29| I292 I303 I304 I3 I6 I324 I325 I326 I328 I335 I355

|460 |462 |464 |467 I542 I685 I836 I843 I844 I850 I909

I9I5 I929205I 2II| 219423482354235624 I9 242024262429243 | 2433

257226012644

Singleton variable sites (four variants): 0

Parsimony informative sites (four variants): 5

Site positions: I334 I84I I845 I9932428

Protein Coding Region assignation: No

This table shows the results for the Polymorphism site test performed on the Rpp8 sequence data. There were 366 polymorphic sites and 2664 total sites used. 
Table 3 DNA Polymorphism Tests

\begin{tabular}{l}
\hline DNA polymorphism \\
\hline Input Data File: C:I...IRpp8_exon.nex \\
Population used:A_and_B_and_C \\
Number of sequences used: 20 \\
Selected region: I-2748 Number of sites: 2748 \\
Total number of sites (excluding sites with gaps / missing data): 2664 \\
Number of polymorphic (segregating) sites, S: 366 \\
Total number of mutations, Eta: 428 \\
Number of Haplotypes, h: 20 \\
Haplotype (gene) diversity, Hd: 1.000 \\
Variance of Haplotype diversity: 0.00025 \\
Standard Deviation of Haplotype diversity: 0.016 \\
Nucleotide diversity, Pi: 0.04005 \\
Sampling variance of Pi: 0.0000024 \\
Standard deviation of Pi: 0.00 I 56 \\
Nucleotide diversity (Jukes and Cantor), Pi(JC): 0.0412 I \\
Theta (per site) from Eta: 0.04529 \\
Theta (per site) from S, Theta-W: 0.03873 \\
Variance of theta (no recombination): 0.0001722 \\
Standard deviation of theta (no recombination): 0.01312 \\
Sariance of theta (free recombination): 0.000004 I \\
\hline
\end{tabular}

\section{Finite sites model}

Theta (per site) from Pi: 0.04231

Theta (per site) from S: 0.04224

Theta (per site) from Eta: 0.04769

Average number of nucleotide differences, $\mathrm{k}$ : 106.689

Stochastic variance of $\mathrm{k}$ (no recombination), Vst(k): $2056.4 \mathrm{I}$ I

Sampling variance of $\mathrm{k}$ (no recombination), $\mathrm{Vs}(\mathrm{k}): 232.418$

Total variance of $\mathrm{k}$ (no recombination), $\mathrm{V}(\mathrm{k}): 2288.829$

Stochastic variance of $\mathrm{k}$ (free recombination), Vst(k): 35.563

Sampling variance of $\mathrm{k}$ (free recombination), $\mathrm{Vs}(\mathrm{k}): 3.743$

Total variance of $\mathrm{k}$ (free recombination), $\mathrm{V}(\mathrm{k}): 39.307$

Theta (per sequence) from S, Theta-W: 103.164

Variance of theta (no recombination): 1221.932

Variance of theta (free recombination): 29.07

This table displays the results from the DNA polymorphism test. Pi was calculated to be 0.04005 , $\mathrm{k}$ was 107, and theta was 103.164.
Table 4 DNA Comparisons

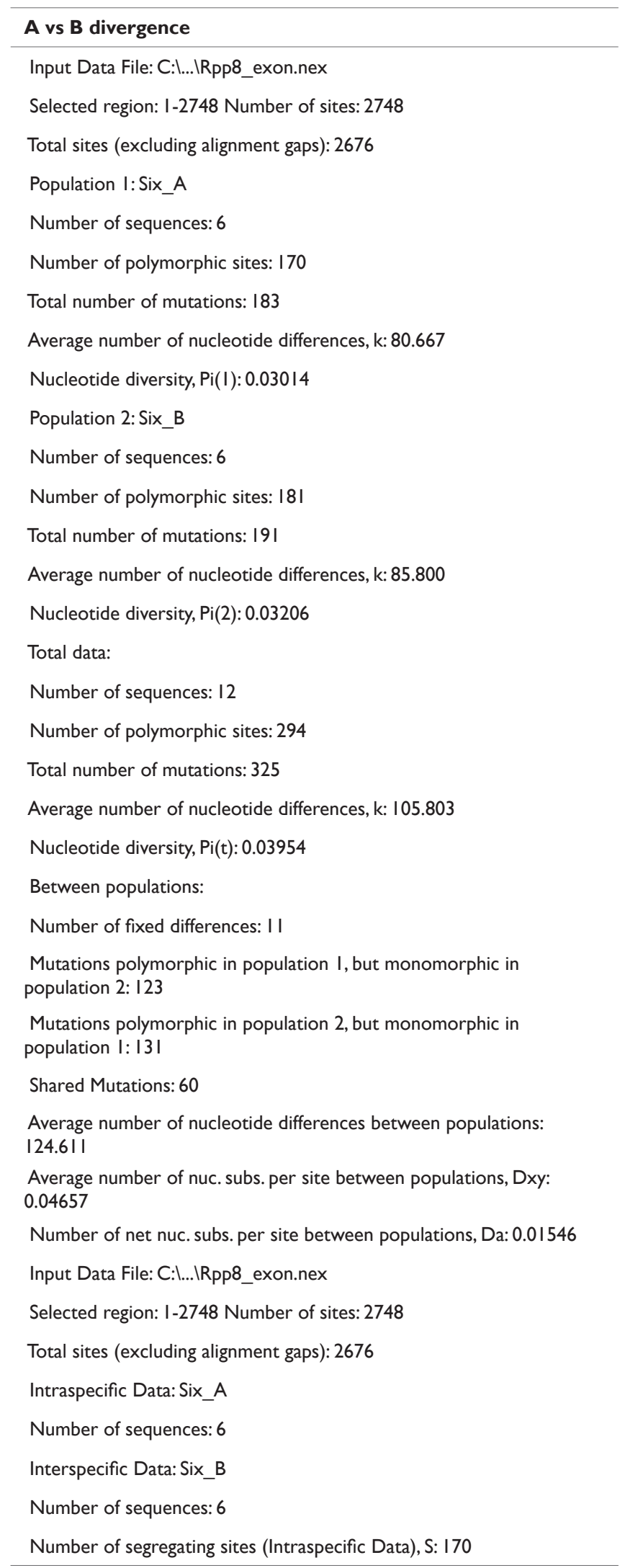


Table Continued..

\begin{tabular}{l}
\hline Analysis of all sites \\
\hline Total number of sites analyzed: 2676 \\
Polymorphism \\
Nucleotide Diversity, Pi (Total): 0.03014 Pi (JC-Total): 0.03077 \\
Theta (Total): 0.02995 Total number of substitutions: 183.00 \\
Divergence \\
Nucleotide Divergence, K (Total): 0.04657 K (JC-Total): 0.04807 \\
A vs C Divergence \\
Input Data File: C:I..IRpp8_exon.nex \\
Selected region: I-2748 Number of sites: 2748 \\
Total sites (excluding alignment gaps): 2703 \\
Intraspecific Data: Six_A \\
Number of sequences: 6 \\
Interspecific Data: Eight_C \\
Number of sequences: 8 \\
Number of segregating sites (Intraspecific Data), S: I72 \\
\hline$========$ Analysis of all sites ========
\end{tabular}

Total number of sites analyzed: 2703

Polymorphism

Nucleotide Diversity, Pi (Total): 0.03009 Pi (JC-Total): 0.0307I

Theta (Total): 0.02997 Total number of substitutions: 185.00

Divergence

Nucleotide Divergence, K (Total): 0.03665 K (JC-Total): 0.03757

Input Data File: C:I...IRpp8_exon.nex

Selected region: I-2748 Number of sites: 2748

Total sites (excluding alignment gaps): 2703

Population I: Six A

Number of sequences: 6

Number of polymorphic sites: 172

Total number of mutations: 185

Average number of nucleotide differences, k: 8I.333

Nucleotide diversity, $\mathrm{Pi}(\mathrm{I}): 0.03009$

Population 2: Eight_C

Number of sequences: 8

Number of polymorphic sites: 279

Total number of mutations: 315

Average number of nucleotide differences, k: 109.357

Nucleotide diversity, $\mathrm{Pi}(2): 0.04046$

Total data:

Number of sequences: 14
Table Continued..

Number of polymorphic sites: 302

Total number of mutations: 352

Average number of nucleotide differences, k: 99.308

Nucleotide diversity, $\mathrm{Pi}(\mathrm{t}): 0.03674$

Between populations:

Number of fixed differences: 0

Mutations polymorphic in population I, but monomorphic in population 2: 37

Mutations polymorphic in population 2, but monomorphic in population I: 167

Shared Mutations: I48

Average number of nucleotide differences between populations: 99.063

Average number of nuc. subs. per site between populations, Dxy: 0.03665

Number of net nuc. subs. per site between populations, Da: 0.00138

$B$ vs $C$ Divergence

Input Data File: C:I...IRpp8_exon.nex

Selected region: I-2748 Number of sites: 2748

Total sites (excluding alignment gaps): 2664

Intraspecific Data: Eight_C

Number of sequences: 8

Interspecific Data: Six_B

Number of sequences: 6

Number of segregating sites (Intraspecific Data), S: 270

=ニニニニニニ= Analysis of all sites =ニニニ=ニニ=

Total number of sites analyzed: 2664

Polymorphism

Nucleotide Diversity, Pi (Total): 0.03980 Pi (JC-Total): 0.04090

Theta (Total): 0.044 I 6 Total number of substitutions: 305.00

Divergence

Nucleotide Divergence, K (Total): 0.04576 K (JC-Total): 0.0472 I

Input Data File: C:I...IRpp8_exon.nex

Selected region: I-2748 Number of sites: 2748

Total sites (excluding alignment gaps): 2664

Population I: Eight_C

Number of sequences: 8

Number of polymorphic sites: 270

Total number of mutations: 305

Average number of nucleotide differences, k: 106.036

Nucleotide diversity, $\mathrm{Pi}(\mathrm{I}): 0.03980$

Population 2: Six_B 
Table Continued...

Number of sequences: 6

Number of polymorphic sites: 177

Total number of mutations: 186

Average number of nucleotide differences, k: 83.267

Nucleotide diversity, $\mathrm{Pi}(2): 0.03126$

Total data:

Number of sequences: 14

Number of polymorphic sites: 346

Total number of mutations: 398

Average number of nucleotide differences, $\mathrm{k}$ : I 10.648

Nucleotide diversity, Pi(t): 0.04153

Between populations:

Number of fixed differences: 5

Mutations polymorphic in population I, but monomorphic in population 2: 207

Mutations polymorphic in population 2, but monomorphic in population I: 88

Shared Mutations: 98

Average number of nucleotide differences between populations: 121.896

Average number of nuc. subs. per site between populations, Dxy: 0.04576

Number of net nuc. subs. per site between populations, Da: 0.01023

This table lists the results from the divergence comparisons that were made on the Rpp8 gene sequences. In the table, there were two sets of tests performed on the data. The first one was a nucleotide-focused divergence test. And the second test is a DNA divergence and convergence statistical analysis. All of the possible comparisons among the three gene sequences were connected.

Table 5 FAHI DNA Polymorphism Test

\section{FAH I}

Input Data File: C:I...Isequence_I.fas

Selected region: I-I563 Number of sites: I563

Total sites (excluding alignment gaps): 912

Intraspecific Data: fhf

Number of sequences: 57

Number of polymorphic (segregating) sites, S: 10

Genetic Code: Nuclear Universal

Protein Coding, and Noncoding Regions analyzed:

Number of protein coding regions (exons): 0

Number of noncoding regions (intronic and flanking regions): I

Non coding region, from site: I to I563
Table Continued..

$====+=+$ Analysis of silent sites $==+===+=$

Total number of codons: 0

Number of codons analyzed: 0 ( 0 sites )

Total number of silent sites (synonymous and noncoding positions): 912.000

Total number of synonymous sites analyzed: 0.000

Total number of noncoding positions analyzed: 912

Polymorphism

Nucleotide Diversity, Pi (Silent): 0.0035I Pi (JC-Silent): 0.00352

Theta (Silent): 0.00238 Number of silent substitutions: 10.00

This was a DNA polymorphism test that was done on the FAHI alleles. The purpose of this test was to find the number of segregating sites in order to complete the HKA calculator.This test only looked at the $57 \mathrm{~A}$. thaliana alleles. It did not include the Aly FAHI allele. We see that the number of segregating sites expressed is 10 .

Table 6 DNA Polymorphism Difference between Random Allele and A. lyrata

\section{Aly vs Loci one}

Input Data File: C:I...IDownloadslcombined.fas

Selected region: I-2748 Number of sites: 2748

Total sites (excluding alignment gaps): 27/2

Population I:Alyrata

Number of sequences: 2

Number of polymorphic sites: 70

Total number of mutations: 70

Average number of nucleotide differences, k: 70.000

Nucleotide diversity, $\mathrm{Pi}(\mathrm{I}): 0.0258 \mathrm{I}$

Population 2: allele_one

Number of sequences: 6

Number of polymorphic sites: 175

Total number of mutations: 188

Average number of nucleotide differences, $\mathrm{k}: 82.733$

Nucleotide diversity, $\mathrm{Pi}(2): 0.0305$ I

Total data:

Number of sequences: 8

Number of polymorphic sites: 384

Total number of mutations: 419

Average number of nucleotide differences, $\mathrm{k}$ : 159.036

Nucleotide diversity, $\mathrm{Pi}(\mathrm{t}): 0.05864$

Between populations:

Number of fixed differences: 165

Mutations polymorphic in population I, but monomorphic in population 2: 66

Mutations polymorphic in population 2, but monomorphic in population I: 184 
Table Continued...

Shared Mutations: 4

Average number of nucleotide differences between populations: 261.833

Average number of nuc. subs. per site between populations, Dxy: 0.09655

Number of net nuc. subs. per site between populations, Da: 0.06839

Aly vs Loci two

Input Data File: C:I...IDownloadslcombined.fas

Selected region: I-2748 Number of sites: 2748

Total sites (excluding alignment gaps): 903

Population I:Alyrata

Number of sequences: 2

Number of polymorphic sites: 24

Total number of mutations: 24

Average number of nucleotide differences, k: 24.000

Nucleotide diversity, $\mathrm{Pi}(\mathrm{I}): 0.02658$

Population 2: alleletwo

Number of sequences: 57

Number of polymorphic sites: 67I

Total number of mutations: 680

Average number of nucleotide differences, k: 310.633

Nucleotide diversity, $\mathrm{Pi}(2): 0.34400$

Total data:

Number of sequences: 59

Number of polymorphic sites: 839

Total number of mutations: I I79

Average number of nucleotide differences, k: 333.99

Nucleotide diversity, $\mathrm{Pi}(\mathrm{t}): 0.36987$

Between populations:

Number of fixed differences: 478

Mutations polymorphic in population I, but monomorphic in population 2: 21

Mutations polymorphic in population 2, but monomorphic in population I: 677

\section{Shared Mutations: 3}

Average number of nucleotide differences between populations: 663.719

Average number of nuc. subs. per site between populations, Dxy: 0.73502

Number of net nuc. subs. per site between populations, Da: 0.54973

This table shows the results from the polymorphism difference test. This was conducted in order to find the difference between these two allele types for the HKA calculator.
Table 6 Results from the Direct HKA Calculator

\begin{tabular}{lll}
\hline HKA test. Direct mode & Locus I & Locus I \\
\hline Interspecific polymorphism data & & \\
Segregating sites (obs) & 366 & 10 \\
Segregating sites (exp) & 234.05 & 141.95 \\
Total number of sites & 2664 & 912 \\
Sample size & 2664 & 912 \\
Interspecific Divergence & & \\
No. differences (obs) & 159 & 334 \\
No. differences (exp) & 290.95 & 202.05 \\
Total number of sites & 2664 & 912 \\
Chromosomal location & Autosome & Autosome \\
X-square value & 81.945 & P: 0.0000 \\
\hline
\end{tabular}

This table shows the results of the direct HKA calculator.The $\mathrm{X}$-square value is $\sim 82$, ad the $\mathrm{P}$-value is $\sim 0$.

\section{Discussion}

From the neighbor-joined phylogeny, there is a significant amount of evidence for interlocus exchange. In the phylogeny, every branch shares significant similarities with other branches within and in different clades. Also, the phylogeny is in two clades. There is one "outlier," WUA, that shares remarkable similarities with the rest of the gene sequences. And the second clade is one big clade that devolves into smaller clades. This de-evolution of the clade design shows significant amount of genetic overlap between the sequences. And in the second clade, it is shown that almost a third of genetic samples are considered to have the same genetic differences. All of these are traits of interlocus exchange. Because of the exchange, the sequences share a significant amount of similarities. Overall, the phylogeny tree suggests sharing or exchange of alleles/polymorphism between the A, $\mathrm{B}$ and $\mathrm{C}$ loci. These similarities influence the shape of the phylogeny, making all of the branches and clades relatively similar.

Around $\sim 5 \%$ of the segregated DNA polymorphism is shared between loci and $95 \%$ is distinct to each locus. This can be done by directly comparing the number of shared and locus-specific segregating sites and/or by comparing the within-and betweenlocus nucleotide diversity. This paper finds this amount by finding the average of pi between all three of the loci comparisons (Table 5). $\mathrm{Pi}$ from $\mathrm{A}$ vs $\mathrm{B}$ is 0.03954 . Pi from $\mathrm{A}$ vs $\mathrm{C}$ is 0.0367 . And $\mathrm{Pi}$ from $\mathrm{B}$ vs $\mathrm{C}$ is 0.04153 . Taking the average and multiplying by 100 , we get a similarity rate of about $5 \%$. And we can find the distinction rate by doing $1-5 \%$, which gives us $95 \%$. There is evidence for interlocus exchange. This is shown by looking at the pi values and the $\mathrm{k}$-values for the nucleotide diversity. All of the pi values for each of the comparisons is between $3-6 \%$ (Table 5). This is significant as this means that $3-6 \%$ of the nucleotides are different when being compared to a chromosomal paralog. As such, this supports the idea that these genes exist within a interlocus exchange. This is because the genes are still mainly similar, while also sharing significant differences. And because of the similarity between all three of these genes, the differences coming from one gene might be coming from both of its chromosomal paralogs. This explains why the pi value 
for all of the genes is somewhat constant, as it characterized by an interlocus exchange. Intergenic exchange is more common between $\mathrm{B}$ and $\mathrm{C}$ loci than it is between any other loci combination. This was obtained by looking at the pi and $\mathrm{k}$ values of all of the possible combinations of loci. Pi from A vs B is 0.03954 . Pi from A vs C is 0.0367. And Pi from B vs C is 0.04153 (Table 4;5) Because of this, $\mathrm{B}$ vs $\mathrm{C}$ pi's is the greatest number. This is significant because that means that $\mathrm{B}$ vs $\mathrm{C}$ have the greatest amount of genetic differences. And since there is evidence that these differences are mainly coming from interlocus exchanges, then this supports the idea that intergenic exchange between the $\mathrm{B}$ and $\mathrm{C}$ loci is more common than any other combination. Otherwise, this particular combination wouldn't have the largest pi value.

However, we must keep in mind that Arabidopsis is normally selffertilizing, so almost all individuals in nature will be homozygous for an identical chromosome. In order to account for this context, we must hypothesize how theoretically ringing intergenic recombination propensities would correlate without date. In line with the data, intergenic recombination propensities would be dictated by the homozygous nature of the chromosomes. This would allow for less diversity in gene sequences and less influential recombination. The recombination propensity would also focus on the $\mathrm{B}$ and $\mathrm{C}$ loci. This is because they are shown to be the center of genetic changes out of the three possible recombination events. As such, the intergenic recombination would share propensities with these two loci. These propensities might include the direction of the recombination and the polarity of the dividing cell. All of these characteristics are influential in the process and serve to change the final recombination. There is elevated polymorphism at Rpp8. From the HKA-calculator the $\mathrm{p}$ value was calculated to be 0.0000 , and the $\mathrm{X}$-squared values was found to be 81.945 (Table 6). Both of the calculation was done relative to the auto some (Table 6). Because the $\mathrm{p}$-value is 0 , this means that the association between the increased polymorphism and the presence of Rpp8 is statistically significant. From the gene sequences discussed here, it seems that these significant values came from too much polymorphism among the loci at nonsynonymous sites. This provides evidence of a positive directional flow, meaning that there is elevated polymorphism at Rpp8. All of the increased of the HKA-calculator to the $\mathrm{p}$-value indicates that there is an elevated polymorphism at Rpp8. This elevated polymorphism has some potential causes. A polymorphism can be maintained by a balance between variation created by new mutations and natural selection. Genetic variation may be caused by frequency-dependent selection. Multiple niche polymorphisms exist when different genotypes should have different fitnesses in different niches. Heterozygous advantage may maintain alleles which would other be selected against. If selection is operating, migration can introduce polymorphism into a population. For this specific population, it is likely that the polymorphism demonstrated in this paper is coming from a continuously maintained balance of variation created by new mutations and natural selection. This seems to fit with the context of the gene sequences, as they are coming from Arabidopsis, which is self-fertilizing. This means that almost all individuals in the sample will be homozygous for an identical chromosome. This allows for there to exist such a delicate balance, thus impacting the polymorphism.

From the neighbor-joined phylogeny, there is a significant amount of evidence for interlocus exchange. Around $\sim 5 \%$ of the segregated DNA polymorphism is shared between loci and $95 \%$ is distinct to each locus. There is evidence for interlocus exchange. Intergenic exchange is more common between $\mathrm{B}$ and $\mathrm{C}$ loci than it is between any other loci combination. There is elevated polymorphism at Rpp8. All of these observations are coming from the hypothesis that the polymorphism demonstrated in this paper is coming from a continuously maintained balance of variation created by new mutations and natural selection. This seems to fit with the context of the gene sequences, as they are coming from Arabidopsis, which is self-fertilizing. Some future directions of research include looking for the cause of the increased polymorphism in Rpp8. Even though this paper shows the link, it is still unknown why this relationship exists with this specific gene. ${ }^{16}$ More work should be done to clarify this relationship and compare it to similar relationships with other genes. Also more work should be done to find molecular evidence for this relationship. An assay should be done in order to account for the entire normal gene irregularities, which would help give support to the hypothesis discussed here today. Also, more work should be done on other species. Doing more work on other species will assist the relationship presented in this paper. And more work should be done on the phylogenetics of Rpp $8 .{ }^{17}$ It would be beneficial to see a detailed look and phylogeny of how the gene has evolved throughout different contexts and how this is reflected in the data. And a final suggestion would be to clarify the intergenic recombination propensities. These were conjectured within this paper based off of data, but more molecular data needs to be shown for this hypothesis.

\section{Acknowledgements}

None.

\section{Conflict of interest}

The author declares there is no conflict of interest.

\section{References}

1. Tamura K, Nei M, Kumar S. Prospects for inferring very large phylogenies by using the neighbor-joining method. Proc Natl Acad Sci USA. 2004;101(30):11030-11035.

2. Olivier Gascuel, Mike Steel. Neighbor-Joining Revealed. Mol Biol Evol. 2006;23(11):1997-2000.

3. Michelmore RW, Meyers BC. Clusters of resistance genes in plants evolve by divergent selection and a birth-and-death process. Genome Res. 1998;8(11):1113-1130.

4. Allen RL, Bittner-Eddy PD, Grenvitte-Briggs LJ, et al. Host-parasite co evolutionary conflict between Arabidopsis and downy mildew. Science. 2004;306(5703):1957-1960.

5. Bergelson J, Dwyer G, Emerson JJ. Models and data on plant-enemy co evolution. Annu Rev Genet. 2001;35:469-499.

6. Bergelson J, Kreitman M, Stahl EA, et al. Evolutionary dynamics of plant R-genes. Science. 2001;292(5525):2281-2285.

7. Librado P, Rozas J. DnaSP v5: A software for comprehensive analysis of DNA polymorphism data. Bioinformatics. 2009;25(11):1451-1452.

8. Stahl EA, Dwyer G, Mauricio R, et al. Dynamics of disease resistance polymorphism at the Rpm1 locus of Arabidopsis. Nature. 1999;400(6745):667-671

9. Ding J, Zhang WL, Jing ZQ, et al. Unique pattern of R-gene variation within populations in Arabidopsis. Molecular Genetics and Genomics. 2007;277:619-629. 
10. Jiang HY, Wang CC, Ping L, et al. Pattern of LRR nucleotide variation in plant resistance genes. Plant Science. 2007;173(2):253-261.

11. Kuang H, Caldwell KS, Meyers BC, et al. Frequent sequence exchanges between homologs of RPP8 in Arabidopsis are not necessarily associated with genomic proximity. Plant J. 2008;54(1):69-80.

12. Rozas J, Ferrer-Mata A, Sánchez-DelBarrio JC, et al. DnaSP 6: DNA Sequence Polymorphism Analysis of Large Datasets. Mol Biol Evol. 2017;34(12):3299-3302.

13. Rozas J. DNA Sequence Polymorphism Analysis using DnaSP. In: Posada D, editor. Bioinformatics for DNA Sequence Analysis; Methods Mol Biol. 2009;537: 337-350.
14. Rozas J, Sánchez-DelBarrio JC, Messeguer X et al. DnaSP, DNA polymorphism analyses by the coalescent and other methods. Bioinformatics. 2003;19(18):2496-2497.

15. Rozas J, Rozas R. DnaSP version 3: an integrated program for molecular population genetics and molecular evolution analysis. Bioinformatics. 1999;15(2):174-175.

16. Rozas J, Rozas R. DnaSP version 2.0: a novel software package for extensive molecular population genetics analysis. Comput Applic Biosci. 1997;13(3):307-311.

17. Rozas J, Rozas R. DnaSP DNA sequence polymorphism: an interactive program for estimating Population Genetics parameters from DNA sequence data. Comput Applic Biosci 1995;11(6):621-625. 